\title{
Intraoperative Ocular Injury
}

National Cancer Institute

\section{Source}

National Cancer Institute. Intraoperative Ocular Injury. NCI Thesaurus. Code C78385.

Damage to the eye during a surgical procedure. 